
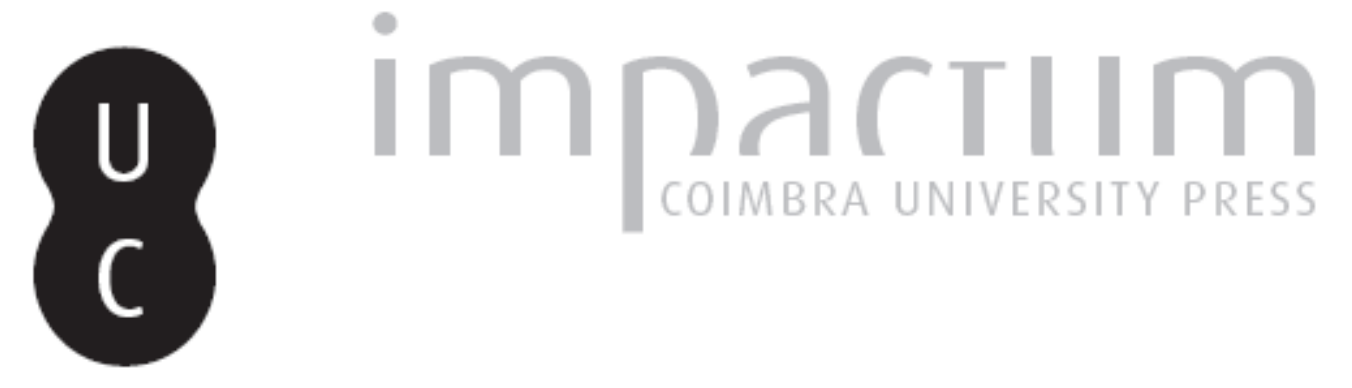

\title{
Análise das condições do ambiente térmico em serviços hospitalares de Medicina física e de reabilitação
}

Autor(es): $\quad$ Rodrigues, C.; Baptista, J. Santos; Brito, M.

Publicado por: Associação Portuguesa de Riscos, Prevenção e Segurança

URL persistente:

URI:http://hdl.handle.net/10316.2/35849

DOI:

DOI:http://dx.doi.org/10.14195/1647-7723_20_16

Accessed : $\quad$ 26-Apr-2023 12:53:02

A navegação consulta e descarregamento dos títulos inseridos nas Bibliotecas Digitais UC Digitalis, UC Pombalina e UC Impactum, pressupõem a aceitação plena e sem reservas dos Termos e Condições de Uso destas Bibliotecas Digitais, disponíveis em https://digitalis.uc.pt/pt-pt/termos.

Conforme exposto nos referidos Termos e Condições de Uso, o descarregamento de títulos de acesso restrito requer uma licença válida de autorização devendo o utilizador aceder ao(s) documento(s) a partir de um endereço de IP da instituição detentora da supramencionada licença.

Ao utilizador é apenas permitido o descarregamento para uso pessoal, pelo que o emprego do(s) título(s) descarregado(s) para outro fim, designadamente comercial, carece de autorização do respetivo autor ou editor da obra.

Na medida em que todas as obras da UC Digitalis se encontram protegidas pelo Código do Direito de Autor e Direitos Conexos e demais legislação aplicável, toda a cópia, parcial ou total, deste documento, nos casos em que é legalmente admitida, deverá conter ou fazer-se acompanhar por este aviso.

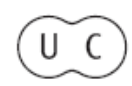




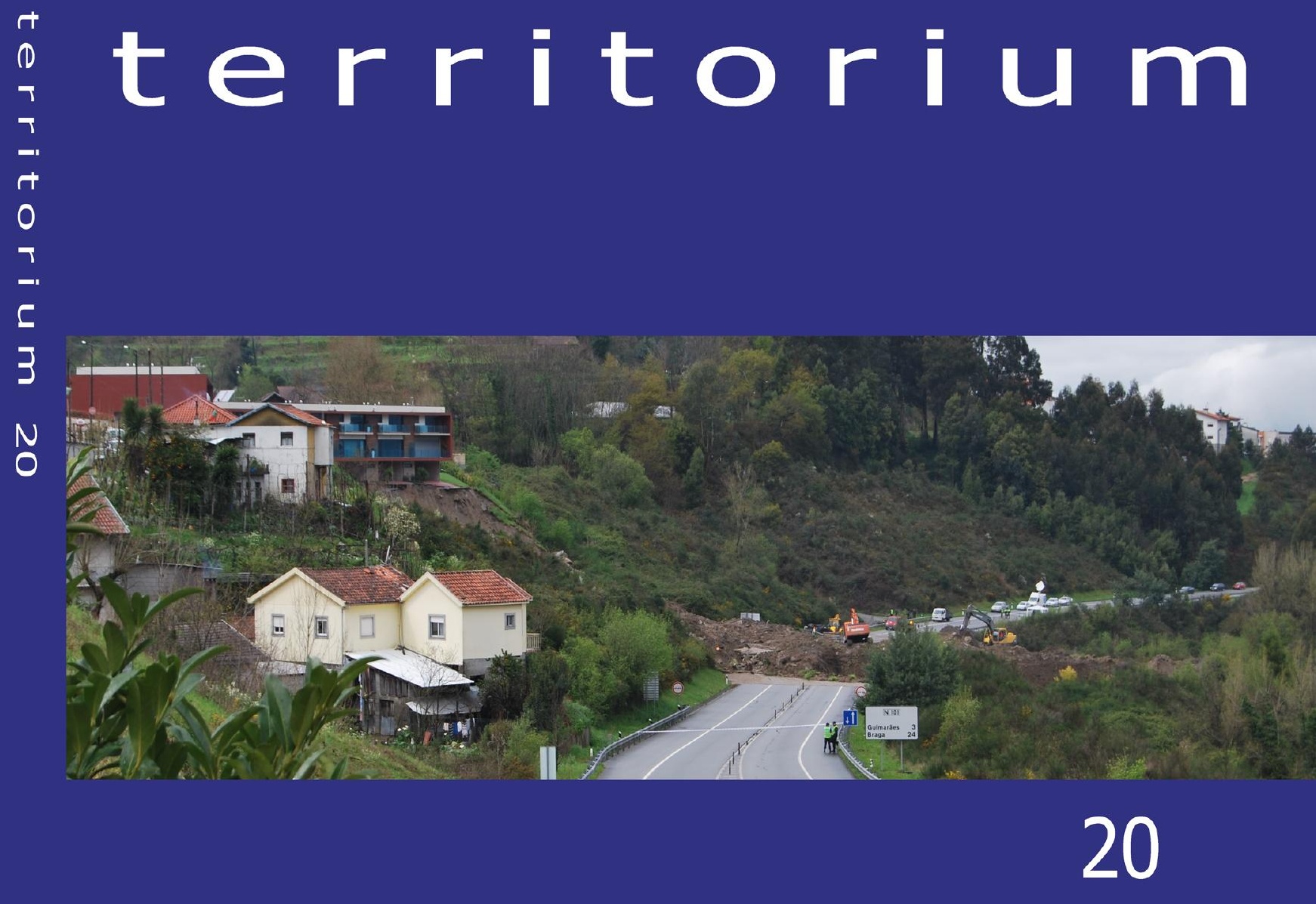

Riscos, População e Segurança 
NATIONAL AERONAUTICS AND SPACE ADMINISTRATION. "The Landsat Program". USA, 2011. Disponível em: http: / / landsat.gsfc. nasa.gov/. Acesso em: 18 abr. 2011.

OECD - Organisation for Economic Co-operation and Development. "Glossary of statstical terms: Land Use”. Paris, 2005. Disponível em: http:// stats.oecd.org/glossary/detail.asp?ID=6493. Acesso em janeiro 2012.

Slater, Philip N. (1980) - "Remote Sensing: optics and optical systems". Addison-Wesley Publishing: Massachusetts.

VAsconcelos, C. H.; Novo (2004) - E. Mapeamento do uso e cobertura da terra a partir da segmentação e classificação de imagens - fração solo, sombra e vegetação derivadas do modelo linear de mistura aplicado a dados do sensor TM/ Landsat5, na região do reservatório de Tucuruí - PA. In: "ACTA AMAZONICA", São José dos Campos v. 34 (3), p. 487-493. Disponível em: http: / /acta.inpa.gov.br/fasciculos/34-3/PDF/ v34n3a14.pdf. Acesso em: 03 jun. 2011.

WIKIPEDIAFOUNDATION. Ficheiro: Parana Municip Ibipora. 2006. Disponível em: http://pt.wikipedia.org/ wiki/Ficheiro:Parana_Municip_lbipora.svg, Acesso em jan.2012.

\section{ANÁLISE DAS CONDIÇÕES DO AMBIENTE TÉRMICO EM SERVIÇOS HOSPITALARES DE MEDICINA FÍSICA E DE REABILITAÇÃO*}

\section{Rodrigues}

PROA/LABIOMEP/CIGAR, Faculdade de Engenharia da Universidade do Porto pee10017@fe.up.pt

J. Santos Baptista

PROA/LABIOMEP/CIGAR, Faculdade de Engenharia da Universidade do Porto jsbap@fe.up.pt

M. Brito

CMUP, Faculdade de Ciências da Universidade do Porto mabrito@fc.up.pt

\section{Introdução}

Um dos fatores de risco físico dos serviços de medicina física e de reabilitação, de qualquer estabelecimento hospitalar é o ambiente térmico, na medida em que profissionais e doentes partilham o mesmo espaço, sujeitos às mesmas condições térmicas, mas com atividade metabólica diversa. Nesta medida, as consequências para uns e outros, quando expostos, no ginásio de reabilitação, às mesmas condições de ambiente térmico é explicada, em grande medida, pela atividade que cada um desenvolve. No caso dos profissionais destes serviços, a temperatura interna pode aumentar, entre outras razões, devido à atividade física e à adoção de posturas exigentes, as quais poderão conduzir a situações de desconforto térmico. Nos doentes, a capacidade física debilitada, associada ao consumo de medicamentos, vão afetar a função metabólica e a perceção de conforto térmico. Temos assim dois grupos para os quais as mesmas condições ambientais podem resultar em consequências diversas.
Nos profissionais, uma situação de desconforto térmico, pode conduzir à adoção de práticas de trabalho inseguras, pelo que poderá haver um aumento da probabilidade de ocorrência de acidentes que será tanto maior quanto maior for o estado de desconforto. Quanto aos doentes, as consequências do desconforto são diferentes. Pela maior dificuldade em controlarem a temperatura interna do corpo, poderão ter mais dificuldade de recuperação e ver, mesmo, agravado o seu estado clínico.

0 presente estudo tem como objetivo analisar as condições do ambiente térmico de alguns ginásios terapêuticos, do serviço de medicina física e reabilitação, de diferentes unidades hospitalares, com vista a identificar os principais parâmetros de variação de conforto térmico e promover a adoção de medidas de conforto térmico seguras, saudáveis e sustentáveis e que possam abranger o maior número, possível, de pessoas.

* O texto desta nota corresponde à comunicação apresentada ao VII Encontro Nacional de Riscos e I Forum ISCIA, tendo sido submetida para revisão em 28-09-2012, e aceite para publicação em 12-11-2013.

Esta nota é parte integrante da Revista Territorium, n. ${ }^{\circ} 20,2013,{ }^{\circ}$ RIscos, ISBN: 0872- 8941. 


\section{Materiais e Métodos}

Foi efetuada uma pesquisa bibliográfica através de uma plataforma online (Metalib), nas seguintes bases de dados: Web of Science; Zentrallblatt MATH Database; PsycArticles; PsycCRITIQUES; PsycBOOKS; Library, Information Science \& Technology; Psychology + Behavior; PsycINFO; PubMed; Regional Business News; MEDLINE; Inspec; Fuente Academica; ERIC; Current Contents; Compendex; Arts \& Humanities Citation Index; Academic Search Complete. A pesquisa foi efetuada por títulos e combinação de pares de palavras relacionadas com o ambiente térmico, em contexto hospitalar. De entre as palavras-chave utilizadas destacam-se: ambiente térmico; conforto térmico; parâmetros de variação térmica; hospitais; serviço de medicina física e de reabilitação.

A recolha de dados decorreu em 2 ginásios de Medicina Física e Reabilitação Hospitalar, durante todo o seu período de funcionamento (média de 8 horas diárias), ao longo 4 dias, em cada um, sem manipulação dos parâmetros de medição, durante os meses de Março e Abril (Primavera). A medição das condições do ambiente térmico foi efetuada com o recurso a um BABUC-A (fig.1) devidamente calibrado. Numa fase posterior foram manipulados dois parâmetros, o vestuário e a exigência da tarefa, de forma a avaliar o respetivo impacto nos resultados.

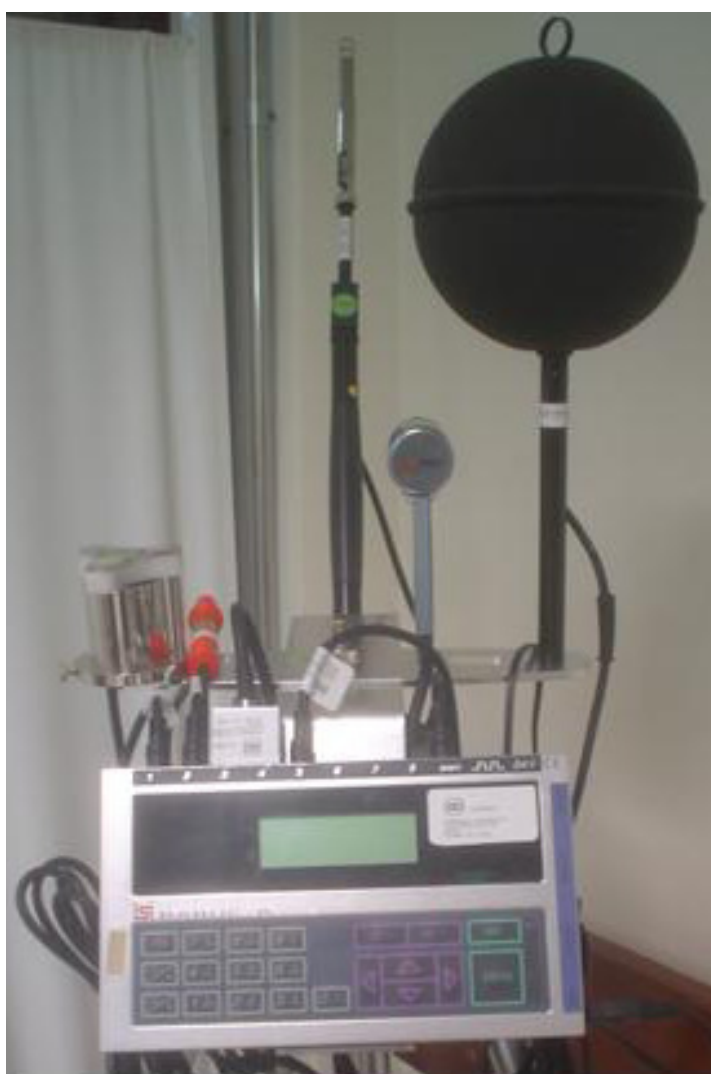

Fig. 1 - BABUC-A.

\section{Revisão Bibliográfica}

Um dos fatores de risco físico dos serviços de medicina física e de reabilitação, de qualquer estabelecimento hospitalar é o ambiente térmico, na medida em que profissionais e doentes partilham o mesmo espaço, sujeito às mesmas condições térmicas, mas em condições diversas.

No caso da grande maioria dos utentes destes ginásios, a variabilidade da resposta resultante da exposição a situações de desconforto térmico é explicada, em grande medida, pela alteração da capacidade de termorregulação, a qual é afetada por inúmeros fatores, entre os quais a idade, devido a uma diminuição da função das glândulas sudoríparas, circulação sanguínea e função cardíaca (KENNEY et MUNCE, 2003), e não apenas pelos fatores diretamente relacionados com o ambiente térmico, como a temperatura, humidade relativa e velocidade do ar.

Para os profissionais, dos serviços de Medicina Física e Reabilitação Hospitalar (Fisioterapeutas, Assistentes Operacionais), a temperatura interna pode aumentar, entre outras razões, devido à adoção de posturas mais exigentes fisicamente (Rodrigues, Guedes et al., 2011), as quais poderão conduzir a situações de desconforto térmico. Para além disso, a exposição a temperaturas elevadas pode levar a alterações cognitivo-comportamentais, tais como, alterações de comportamento, distúrbios de ansiedade e depressão (TAWATSUPA et al., 2010), aumentando, assim, a probabilidade de ocorrência de acidentes, com o avanço do estado de fadiga. A temperatura, por sua vez, influencia positivamente o processo de acumulação de fadiga, sendo que a prática de esforços em ambientes quentes ou muito frios reduzem a capacidade de trabalho (Rodrigues, Guedes et al., 2011) e diminuem a capacidade de vigília e a resistência, em particular no caso de exposição ao calor (ENANDER, 1989).

No caso dos doentes, a capacidade física debilitada, associada ao consumo de medicamentos, afeta a sua perceção de conforto térmico, o que os torna num grupo de risco. Nesse sentido o controlo da respetiva temperatura interna é essencial para evitar a deterioração do seu estado clínico. No caso de crianças, idosos, gestantes, pessoas com limitações físicas e cognitivas e também as de baixo poder socioeconómico, dado fazerem parte dos principais grupos de risco (Balbus et Malina, 2009), a atenção no controlo da temperatura interna deve ser redobrada, quando se encontram doentes.

O quadro legal existente (TABELA I), define algumas condições em que estas atividades devem recorrer, no sentido de garantir a qualidade mínima do ar a que 
TABELA I - Referenciais Normativos.

\begin{tabular}{|c|l|l|}
\hline \multicolumn{1}{|c|}{ Legislação } & $\begin{array}{l}\text { Portaria n. }{ }^{\circ} 1212 / 2010 \\
\text { (Unidades Privadas) }\end{array}$ & $\begin{array}{c}\text { Temperatura (Ginásios): } \\
\text { Verão - máximo: } 25^{\circ} \mathrm{C} \\
\text { Inverno - mínimo: } 20^{\circ} \mathrm{C}\end{array}$ \\
\hline Referenciais Técnicos & ACSS (2008) & $\begin{array}{c}\text { Temperatura (local não especificado): } \\
\text { Verão - máximo: } 25^{\circ} \mathrm{C} \\
\text { Inverno - mínimo: } 20^{\circ} \mathrm{C}\end{array}$ \\
\hline Legislação & Decreto-Lei n. ${ }^{\circ}$ 243/86 (Estabelecimentos comerciais, escritórios e serviços) & $50-70 \%$ \\
\hline Normas & ISO 7730 & $\begin{array}{c}\text { Conforto térmico: } \\
\text { PMV }[-0,5 ; 0,5] \\
\text { PPD }<10 \%\end{array}$ \\
\hline
\end{tabular}

profissionais e doentes estão expostos. No entanto, nem sempre atende às necessidades particulares de cada binómio doente / profissional.

\section{Resultados e Discussão}

\section{Condições reais não manipuladas}

Os resultados da medição, efetuada no Ginásio $A$, demostram valores de desconforto térmico dos fisioterapeutas nos 4 dias de medição, com um valor de PMV superior 0,5 (fig. 2), indo ao encontro dos valores de PPD superiores a $10 \%$, ao longo de todo o dia de trabalho (fig. 3).

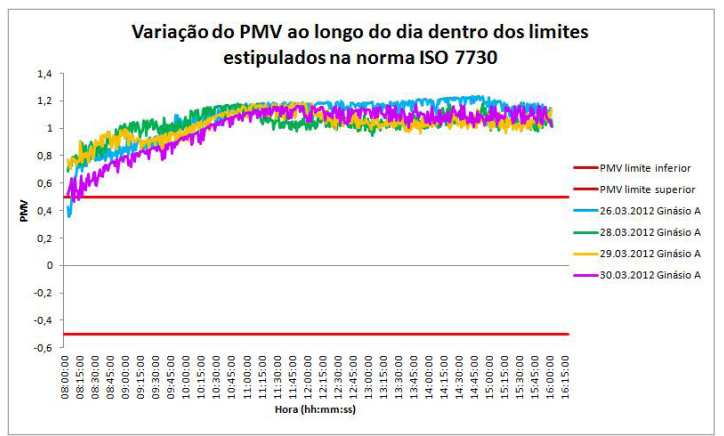

Fig. 2 - PMV Ginásio A.

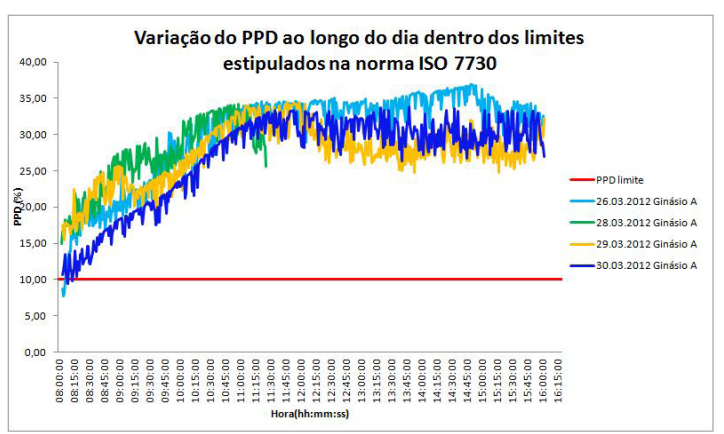

Fig. 3 - PPD Ginásio A.

No Ginásio B (Fig. 4 e 5), dos 4 dias de medição, 3 apresentam valores de PMV superiores a 0,5 e valores de PPD superiores a $10 \%$, sendo que apenas num dos dias se registam valores de conforto térmico (PMV entre -0,5 e $0,5$ e PPD $<10 \%)$.

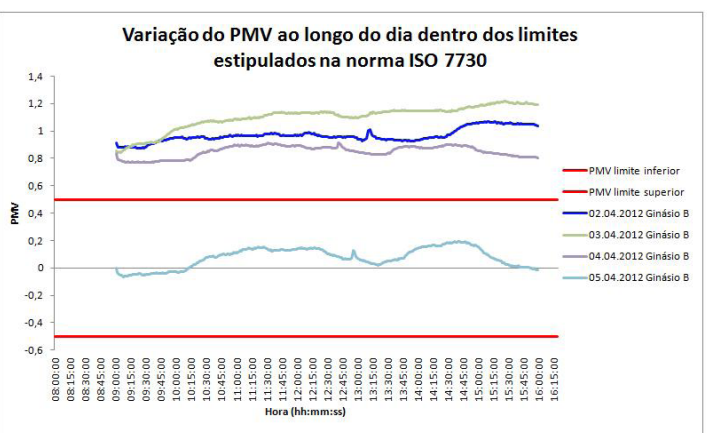

Fig. 4 - PMV Ginásio B.

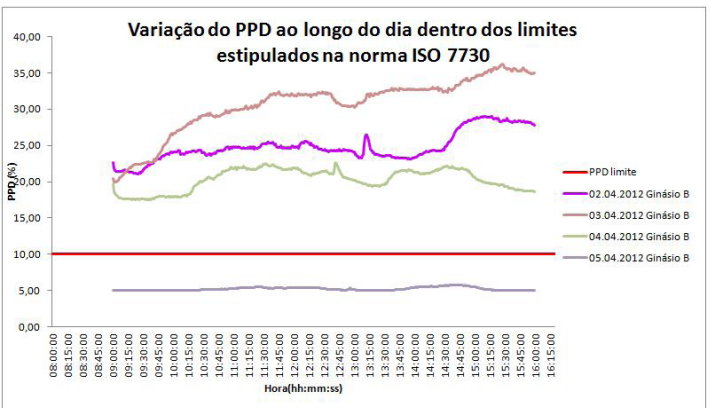

Fig. 5 - PPD Ginásio B.

Condições manipuladas (vestuário e a exigência da tarefa)

Tendo-se verificado que a generalidade dos valores registados se encontrava fora da zona de conforto, partiu-se para a simulação para condições distintas das efetivamente verificadas, no sentido de se verificar possibilidade de melhorar as condições de conforto dos profissionais. Essa simulação foi efetuada utilizando os dados ambientais medidos, mas reduzindo os níveis de isolamento proporcionados pelo vestuário e a atividade física dos profissionais. Como resultados conseguiram-se obter valores para o PMV e para o PDD dentro dos limites de conforto térmico, tanto no Ginásio A (fig. 6 e 7), como no Ginásio B (fig. 8 e 9).

\section{Conclusão}

As condições térmicas nos 2 ginásios avaliados apresentaram valores fora dos limites de conforto térmico estabelecidos pela Norma ISO 7730. Tais valores resultam, em grande medida, de valores de humidade relativa muito baixos, os quais associados à grande 


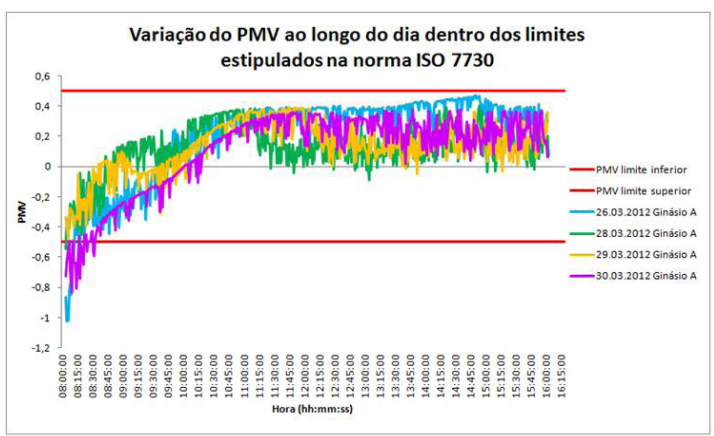

Fig. 6 - PMV (manipulado) Ginásio A.

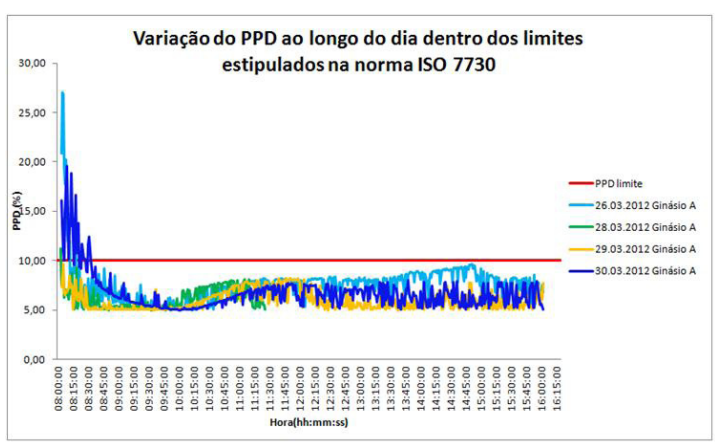

Fig. 7 - PPD (manipulado) Ginásio B.

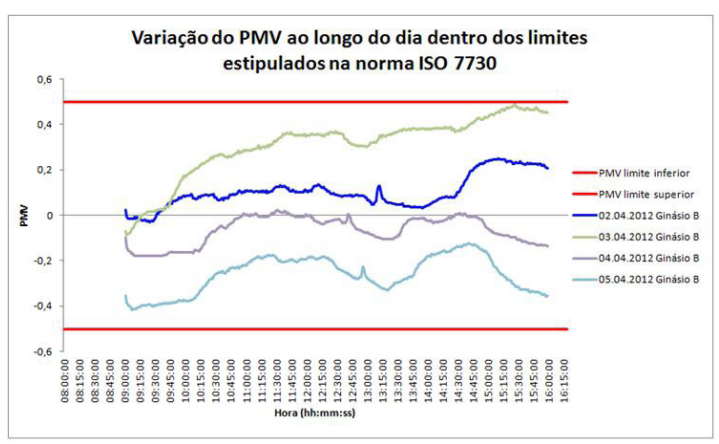

Fig. 8 - PMV (manipulado) Ginásio B.

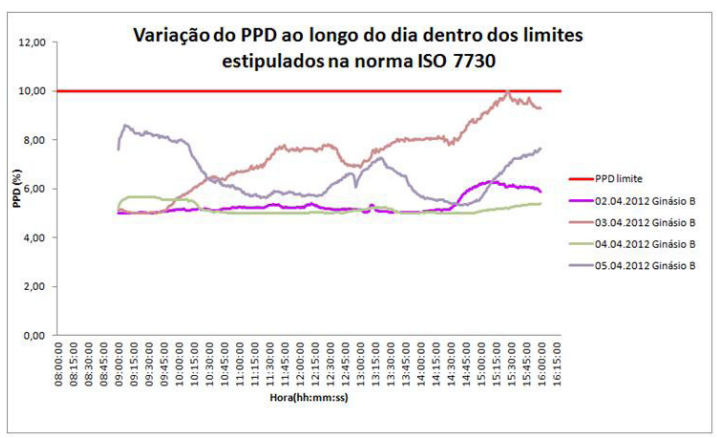

Fig. 9 - PPPD (manipulado) Ginásio B. exigência em termos de atividade física e postural e também ao uso de demasiadas peças de vestuário, acabam por potenciar a sensação de desconforto.

Amanipulação, por simulação, dos parâmetros relativos ao vestuário e ao metabolismo (exigência da tarefa) revelou a possibilidade de obtenção de resultados satisfatórios em termos de conforto térmico a partir de medidas preventivas simples e sustentáveis como, por exemplo, alteração do vestuário e a rotatividade de tarefas.

\section{Referências bibliográficas:}

ACSS (2008) - Especificações Técnicas para a Instalação de AVAC. ET 06/2008. V 2010: 10 - 25. Administração Central do Sistema de Saúde.

Balbus, J. M. et C. Malina (2009). "Identifying Vulnerable Subpopulations for ClimateChange HealthEffects in the United States." Journal of occupational and environmental medicine 51(1): 33-37.

DECRETO-LEI (N. ${ }^{\circ}$ 243/86). “Decreto-Lei n. ${ }^{\circ} 243 / 86$, de 20 de agosto.” Diário da República n. 190. I Série: 2099 - 2106.

EnANDER, A. E. (1989). "Effects of thermal-stress on human-performance." Scandinavian journal of work, environment \& health 15: 27-33.

ISO 7730: 2005. "Ergonomics of the thermal environment: Analytical determination and interpretation of thermal comfort using calculation of the PMV and PPD indices and local thermal comfort criteria."

Kenney, W. L. et T. A. Munce (2003). "Invited review: Aging and human temperature regulation." Journal of applied physiology 95(6): 2598-2603.

PORTARIA (N. ${ }^{\circ}$ 1212/2010). "Portaria n. ${ }^{\circ} 1212 / 2010$, de 30 de novembro." Diário da República n. ${ }^{\circ} 232$. I Série: 5409 - 5418.

Rodrigues, C. , Guedes, J. C. et J. S. Baptista (2011). "Ambiente Térmico e o seu impacto do Homem - Análise numa prespectiva Homem/Ambiente/ Trabalho." Congresso Luso-Moçambicano de Engenharia.

Tawatsupa, B., LiM, L. L. Y., Kuellstrom, T., Seubsman, S. -A, ET A. SLEIGH (2010). "The association between overall health, psychological distress, and occupational heat stress among a large national cohort of 40,913 Thai workers." Global Health Action 3: 1-10. 